\title{
Influence des pratiques agricoles sur la macrofaune du sol : cas de l'enfouissement de la paille et du fumier
}

\author{
Mamoudou TRAORE ${ }^{1 *}$, François LOMPO ${ }^{2}$, Frederic AYUKE ${ }^{3}$, Badiori OUATTARA ${ }^{2}$, \\ Korodjouma OUATTARA ${ }^{4}$ et Michel SEDOGO ${ }^{2}$ \\ ${ }^{1}$ Institut de l'Environnement et de Recherches Agricoles (INERA), Département Production Forestières 03 BP \\ 7047 Ouagadougou 03 Burkina Faso. Tél. : 226503340 98. Fax : 22650340271. \\ ${ }^{2}$ Institut de l'Environnement et de Recherches Agricoles (INERA), Laboratoire Sol-Eau-Plantes. 04 BP 8645 \\ Ouagadougou 04, Burkina Faso. Tél. : 00226503402 70. Fax. : 0022650340271. \\ ${ }^{3}$ Department of Land Resources \& Agricultural Technology (LARMAT), College of Agriculture \& Veterinary \\ Medicine, University of Nairobi, P.O Box 30197-00100, Nairobi, Kenya. \\ ${ }^{4}$ Institut de l'Environnement et de Recherches Agricoles (INERA), Station de Recherche de Saria, Laboratoire \\ Sol-Eau-Plantes, BP 10 Koudougou Burkina Faso. Tel. : 22650446509. \\ *Auteur correspondant, E-mail : tramadalbela@yahoo.fr
}

\section{RESUME}

La macrofaune du sol sur un essai agricole de longue durée a été inventoriée en 2008, huit semaines après les semis. Cet essai agricole implanté en 1980 est situé au Centre Ouest du Burkina Faso, et est représentatif de la production du sorgho avec utilisation de deux types de matières organiques exogènes avec ou sans fertilisation azotée. L'objectif était d'étudier l'influence de ces pratiques agricoles sur la macrofaune des sols, représentée par les termites et les vers de terre. Les termites ont été échantillonnés par la méthode des monolithes et par transect ; les vers de terre ont été échantillonnés uniquement par monolithes. Sept espèces de termites de la famille des termitidae et trois espèces de vers de terre de la famille des Acanthodrilidae ont été identifiées. L'apport des matières organiques exogènes s'est traduit par une augmentation du nombre d'individus par unité de surface pour les deux groupes de macrofaune. L'incorporation de la paille a été beaucoup plus favorable aux termites par rapport au fumier qui a été plus favorable aux vers de terre. Pour les deux types de matières organiques, l'apport d'azote a entraîné une augmentation du nombre d'individus par unité de surface. La macrofaune dans la jachère bordant le site a été plus diversifiée que dans les sites de culture.

(C) 2012 International Formulae Group. All rights reserved.

Mots clés: macrofaune du sol, fertilisation, paille, fumier, jachère.

\section{INTRODUCTION}

La diminution progressive de la biodiversité et l'appauvrissement des terres agricoles sont une préoccupation mondiale car $76 \%$ des terres cultivées dans le monde souffrent d'une dégradation qui peut aller de légère à très sévère (Bot et al., 2000). Au Burkina Faso, comme dans beaucoup de pays de la zone sahélienne, ce phénomène se traduit par un abandon du système traditionnel. On assiste en effet à un raccourcissement de la durée de la jachère ou 
à la culture continue en particulier dans le Centre du pays. La conséquence de ces changements dans l'utilisation des terres agricoles est la baisse des niveaux de fertilité des terres et celle de la production (Ouédraogo, 2005). Cette réalité alarmante interpelle la recherche scientifique pour le développement d'outils de gestion des terres agricoles permettant de prévoir les implications écologiques des pratiques agricoles et leurs conséquences sur le maintien de la stabilité structurale du sol. L'enjeu étant de pouvoir produire plus, de façon durable ; de trouver des alternatives à la jachère longue; de proposer des systèmes de cultures originaux adaptés aux spécificités climatiques et pédologiques des zones soudano-sahéliennes (Sedogo, 1993; Lompo, 2009). Les aléas climatiques, l'inadéquation des pratiques culturales, la pauvreté des sols et la faible fertilisation sont soulignés comme les contraintes majeures à la production agricole au Burkina Faso (Sedogo, 1993; Bonzi, 2002 ; Hien, 2004).

Parmi les actions qui concourent à la protection du sol, un grand nombre d'auteurs attribuent un rôle prépondérant aux invertébrés du sol qui sont des indicateurs très sensibles de la qualité des sols et de leur fertilité (Bachelier, 1978 ; Black et al., 1997; Lavelle et al., 2006). Ainsi, la non-durabilité générale actuelle des systèmes agricoles mondiaux provient, au moins en partie, de la disparition des invertébrés du sol (Lavelle et al., 2006). Parmi ces invertébrés, les organismes ingénieurs du sol, termites et vers de terre, jouent un rôle de conditionneurs du sol par leurs actions de bioturbation et leurs effets sur les paramètres physiques du sol (Bachelier, 1978). Cependant, si plusieurs auteurs ont fait remarquer que l'activité de la faune dans les sols agricoles dépend fortement des pratiques de gestion, ils soulignent toutefois que les facteurs contrôlant l'abondance et la diversité sur cette faune dans les agro-écosystèmes doivent être mieux compris (Marinissen, 1992; Black et al., 1997). Les données comparatives sur la diversité et l'activité de termites sous différents systèmes de culture, en particulier les systèmes traditionnels sont rares et souvent contradictoires et il y a très peu d'études de l'impact des pratiques de gestion des terres agricoles sur la faune des sols.

Dans les écosystèmes tropicaux et subtropicaux, la macrofaune est essentiellement représentée par les termites, les fourmis et les vers de terre, avec des abondances respectives de $37 \%, 23 \%$ et $9 \%$ (Fragoso et al., 1995). Dans ces écosystèmes, les pratiques agricoles qui portent atteinte à la macrofaune sont à éviter car pour la plante du sorgho (Sorghum bicolor (L.) Moench, 1794) par exemple, une économie d'eau et une utilisation efficiente des ressources organiques ont été constatées en présence de la macrofaune (Ouédraogo et al., 2006).

Dans notre étude, les représentants de la macrofaune considérés sont les termites (Isoptères) et les vers de terre (Annélides, Oligochètes). L'objectif de cette étude est d'inventorier la macrofaune du sol sous différents modes de gestion des terres agricoles (apport de matières organiques exogènes et fertilisation minérale) et d'établir une corrélation entre types de matières organiques et de fertilisation apportés et la composition de la macrofaune du sol.

Notre étude a eu pour hypothèse que les pratiques agricoles telles que l'apport de matières organiques exogènes comme le fumier et la paille avec ou sans fertilisation minérale influencent la composition de la macrofaune du sol.

\section{MATERIEL ET METHODES Description du site}

Cette étude s'est déroulée en 2008 sur un essai agricole situé dans la station de recherches environnementales et agricoles de Saria $\left(12^{\circ} 16^{\prime} \mathrm{N}\right.$ et $2^{\circ} 9^{\prime}$ W) au Centre-Ouest du Burkina Faso. Cet essai est nommé Essai Étude Comparative et est situé dans une région soumise à un climat de type soudanien caractérisé par l'existence de deux saisons très marquées : une longue saison sèche (octobre à 
mai) et une courte saison de pluie (juin à octobre). L'altitude de cette station est de 300 $\mathrm{m}$. La température moyenne annuelle est de $28{ }^{0} \mathrm{C}$. Les précipitations moyennes annuelles sont de $800 \mathrm{~mm}$ et son sol est classé dans la catégorie des sols ferrugineux tropicaux lessivés (Hien, 2004).

\section{Description de l'essai agricole}

Ce dispositif a été implanté en 1980 pour comparer les effets de diverses matières organiques exogènes (fumier, paille de sorgho, compost aérobie, compost anaérobie), en présence ou non de fumure azotée (sous forme d'urée) sur les rendements du sorgho et la fertilité du sol. Les parcelles mesurent 5,20 $\mathrm{m}$ de longueur sur $4 \mathrm{~m}$ de largeur. Une allée de $2 \mathrm{~m}$ sépare deux blocs voisins. La dimension du site est de 45,2 $\mathrm{m}$ x $44 \mathrm{~m}=$ $1988,8 \mathrm{~m}^{2}$. C'est un dispositif en factoriel $5 \mathrm{x}$ 2 avec six répétitions. Les dix traitements pratiqués à ce système de culture de sorgho sont :

T1 : Témoin - azote- : « T (-N)»;

$\mathrm{T} 2$ : Témoin +azote- : « $\mathrm{T}(+\mathrm{N}) »$;

$\mathrm{T} 3$ : Compost anaérobie $\left(10 \mathrm{t}^{\mathrm{h}} \mathrm{ha}^{-1} \cdot \mathrm{an}^{-1}\right)$ sans azote- : «Can $(-\mathrm{N}) »$;

T4 : Compost anaérobie (10 t.ha $\left.{ }^{-1} \cdot \mathrm{an}^{-1}\right)+$ azote- : « Can $(+\mathrm{N}) »$;

T5 : Compost aérobie $\left(10 \mathrm{t} \mathrm{ha}^{-1} \mathrm{an}^{-1}\right)$ sans azote- : « Cae $(-\mathrm{N}) »$;

T6 : Compost aérobie $\left(10\right.$ t.ha $\left.^{-1} \cdot \mathrm{an}^{-1}\right)+$ azote -: « Cae $(+\mathrm{N}) »$;

T7 : Fumier $\left(10\right.$ t.ha $\left.{ }^{-1} \cdot \mathrm{an}^{-1}\right)$ sans azote- : «F (N) ;

T8 : Fumier $\left(10\right.$ t.ha $\left.^{-1} \cdot \mathrm{an}^{-1}\right)+$ azote- : $\ll \mathrm{F}$ $(+\mathrm{N}) \gg$;

T9 : Paille (10 t.ha $\left.{ }^{-1} . \mathrm{an}^{-1}\right)$ sans azote- : «P ($\mathrm{N}) »$;

T10 : Paille (10 t.ha $\left.{ }^{-1} \cdot \mathrm{an}^{-1}\right)+$ azote- : $\ll \mathrm{P}$ $(+\mathrm{N}) »$.

L'apport de l'azote est de $60 \mathrm{~kg} \cdot \mathrm{ha}^{-1} \cdot \mathrm{an}^{-1}$ sous forme d'urée $(46 \%$ de $\mathrm{N})$ en deux fractions : une moitié au semis et une autre à la montaison (30 jours après semis). Le compost aérobie est obtenu par le compostage de paille de sorgho et de déjections de bœufs dans des fosses pendant 3 mois. Il est préparé dans des compostières de $1,5 \mathrm{~m}^{3}$. Ce que l'on nomme compost anaérobie dans cette expérimentation est en fait un sous produit de méthanisation issu de la fermentation dans des cuves de biogaz anaérobie de la station; la paille de sorgho et les déjections animales sont fermentées en milieu anaérobie pendant 5 à 6 mois. Les traitements pris en compte dans la présente étude sont les traitements $\mathrm{T}(-\mathrm{N}), \mathrm{T}$ $(+\mathrm{N}), \mathrm{P}(-\mathrm{N}), \mathrm{P}(+\mathrm{N}), \mathrm{F}(-\mathrm{N})$ et $\mathrm{F}(+\mathrm{N})$.

\section{Échantillonnage de la macrofaune}

L'échantillonnage des termites a été effectué huit semaines après les semis par la méthode standard TSBF. Le monolithe est prélevé comme un bloc du sol de $25 \mathrm{~cm}$ x 25 $\mathrm{cm} \times 30 \mathrm{~cm}$ de profondeur (Anderson et al., 1993). Pour l'étude des termites 18 monolithes (3 par traitement) sur l'essai agricole et 3 dans la jachère de bordure du site. Les termites ont été recueillies par tri à la main sur un plateau en plastique. Une fouille complémentaire est réalisée pour la recherche des termites dans un transect de 5 x $2 \mathrm{~m}$ réalisé à côté du monolithe (Jones et al., 2000). Le transect a été fouillé en surface et à une profondeur de $5 \mathrm{~cm}$ à la daba et les termites ont été collectés dans des flacons de conservation. Les échantillons de termites ont été conservés dans des flacons contenant de l'alcool à $75 \%$ et acheminés au laboratoire pour l'identification et la détermination de la biomasse. Les termites ont été déterminés au Département de Zoologie des Invertébrés du Musée National de Nairobi au Kenya et au Laboratoire d'Histoire Naturelle (Centre National de la Recherche Scientifique et Technologique, Burkina Faso).

Les vers de terre ont été échantillonnés uniquement par monolithes. Ils ont été tués dans $75 \%$ d'alcool, puis fixés à $4 \%$ de formaldéhyde. Ils ont été déterminés au 
Département de zoologie des invertébrés du Musée National de Nairobi au Kenya. Comme pour les termites, vingt un (21) monolithes ont été prélevés.

\section{Analyse des données}

Les données obtenues ont été introduites dans le tableur Excel et soumises à l'analyse de variance (ANOVA), General Linear Model (GLM) en utilisant Minitab version 13.0. La matrice d'analyse est un "full rank" constituée des facteurs (type de culture et traitements) et des variables (espèces de termites ou de vers de terre). Pour la description de la macrofaune des sites, plusieurs paramètres ont été utilisés. La diversité spécifique a été exprimée par l'Indice de Shannon : H' $=-\Sigma$ (pi ln pi), où $\mathrm{H}^{\prime}$ : indice de biodiversité de Shannon, $i$ : une espèce du milieu d'étude, $p_{i}$ : proportion d'une espèce $i$ par rapport au nombre total d'espèces (S) dans le milieu d'étude (ou richesse spécifique du milieu), qui se calcule de la façon suivante: $p(i)=n_{i} / N$ où $n_{i}$ est le nombre d'individus pour l'espèce $i$ et $\mathrm{N}$ est l'effectif total (les individus de toutes les espèces) (Magurran, 1988). Un paramètre d'ordre quantitatif, l'abondance a été utilisée pour exprimer le nombre d'individus d'une espèce par unité de surface. Enfin, la biomasse, masse totale de la substance vivante présente sur une unité de surface, a été utilisée pour exprimer l'importance des différentes espèces sur les sites.

\section{RESULTATS}

Au total, sept (07) espèces de termites et trois (03) espèces de vers de terre ont été identifiées sur l'essai agricole et dans les jachères. Pour les termites ce sont: Macrotermes subhyalinus Rambur 1842, Amitermes stephensoni Harris 1957, Microcerotermes parvulus Sjostedt 1911, Odontotermes magdalenae Grassé \& Noirot 1950, Odontotermes sp Holmgren 1912, Microtermes sp Wasmann 1902,
Trinervitermes sp Holmgren 1912. Les espèces de vers de terre identifiées sont: Millsonia inermis Beddard 1894, Dichogaster affinis Michaelsen 1890 et Dichogaster sp Beddard 1888.

Les traitements incluant la paille renferment plus d'individus de termites, suivi des traitements incluant le fumier et le traitement témoin. L'indice de Shannon évolue dans le même sens que le nombre d'individus. Les termites ailés et l'espèce $O$ magdalenae sont les plus nombreux au sein de tous les traitements. La jachère est plus diversifiée que le site de culture avec l'apparition d'espèces lignivores ( $M$. subhyalinus, A. stephensoni et $M$. parvulus). Les valeurs de la biomasse et de l'Indice de Shannon évoluent dans le même sens. Au sein d'un même traitement, l'apport de l'azote s'est traduit par une hausse de diversité à l'exception du traitement témoin (Tableau 1). Le nombre d'individus de termites (toutes les espèces inclues) par $\mathrm{m}^{2}$ est plus élevé dans les traitements avec incorporation de paille. La jachère renferme plus d'individus, suivis du traitement avec paille et du traitement avec fumier. Le traitement témoin renferme peu d'individus et est moins diversifié que les deux autres traitements (Figure 1). Les valeurs de la biomasse sont également plus élevées dans les traitements avec utilisation de paille (Figure 2). Par rapport à l'Indice moyen de Shannon (IS moyen $=0,8035$ ), seul le traitement témoin est situé en dessous de cette valeur (Figure 3). L'analyse statistique (ANOVA) des effets des types de traitements sur les termites montre que deux espèces de termites $O$. magdalenae et $O$. $s p$. sont significativement corrélées au type de traitement avec respectivement des valeurs de $\mathrm{p}=0,027$ et $\mathrm{p}=0,011$ (Tableau 2)

La population de vers de terre est représentée par les deux (02) espèces $M$. inermis et $D$. affinis. L'indice de Shannon est nul pour les traitements témoins, $\mathrm{P}(-\mathrm{N})$ et $\mathrm{F}(-$ 
N). Pour les vers de terre également, l'apport de l'azote a augmenté la diversité sauf pour le témoin (Tableau 3). Le nombre de vers de terre par $\mathrm{m}^{2}$ montre que le traitement avec incorporation du fumier est plus riche en vers de terre par rapport à la paille. La jachère renferme plus d'individus par rapport au site de culture (Figure 4). La biomasse des vers de terre est également plus importante dans le cas d'utilisation du fumier par rapport à la paille
(Figure 5). Par rapport à l'Indice Shannon moyen (IS moyen $=0,2865$ ), seuls les traitements $\mathrm{P}(+\mathrm{N}), \mathrm{F}(-\mathrm{N})$ et la jachère ont des valeurs situées au dessus de cette valeur (Figure 6). L'analyse statistique (ANOVA) des effets des types de traitements sur les vers de terre montre qu'aucune des espèces n'est significativement corrélée au type de traitement avec respectivement des valeurs de $\mathrm{p}=0,206, \mathrm{p}=0,187$ et $\mathrm{p}=0,419$ (Tableau 4).

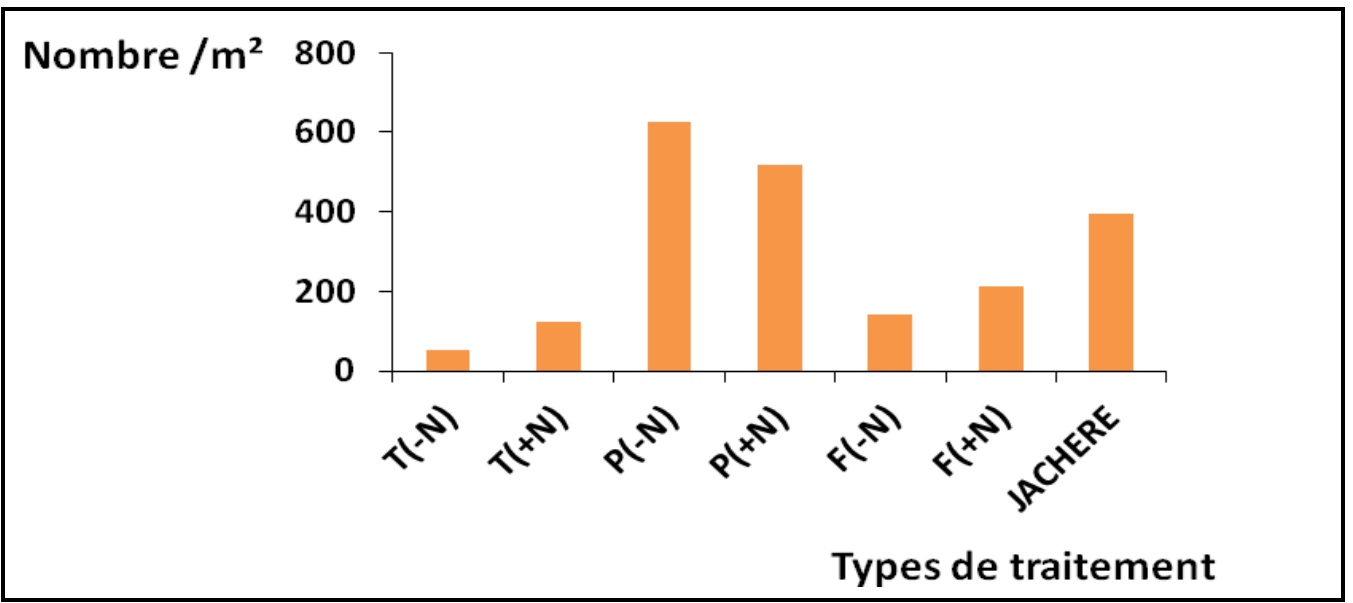

$\mathrm{T}(-\mathrm{N})$ : Témoin sans azote ; $\mathrm{T}(+\mathrm{N})$ : Témoin plus azote ; $\mathrm{F}(-\mathrm{N})$ : Fumier sans azote ; F $(-\mathrm{N})$ : Fumier plus azote ; $\mathrm{P}(-\mathrm{N})$ : Paille sans azote; $\mathrm{P}(-\mathrm{N})$ : Paille plus azote.

Figure 1: Nombre des termites par unité de surface des différents traitements.

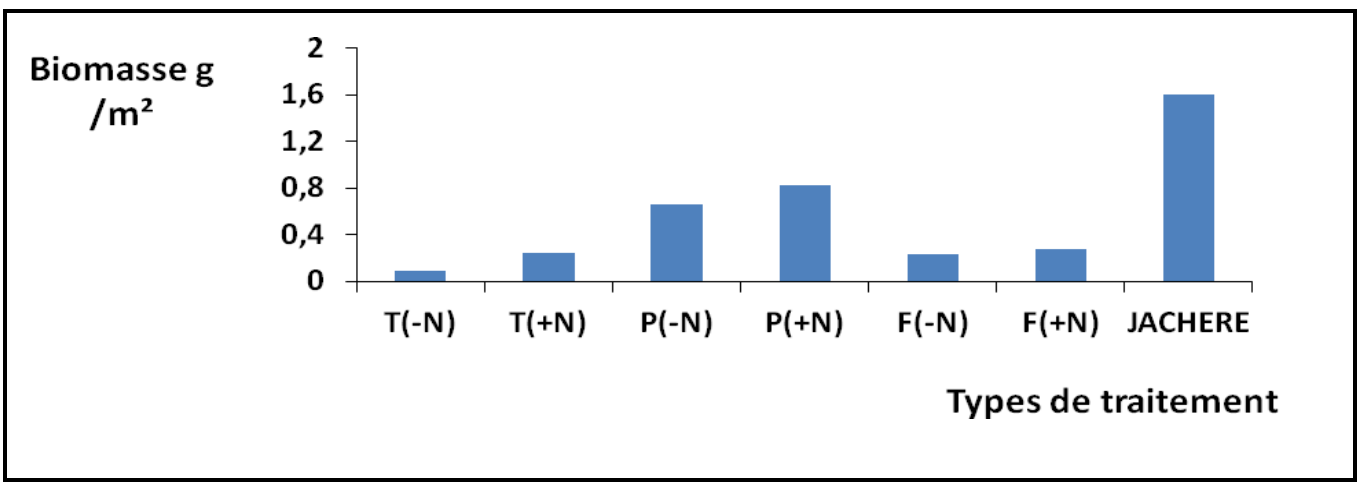

Figure 2 : Biomasse des termites par unité de surface des différents traitements. 


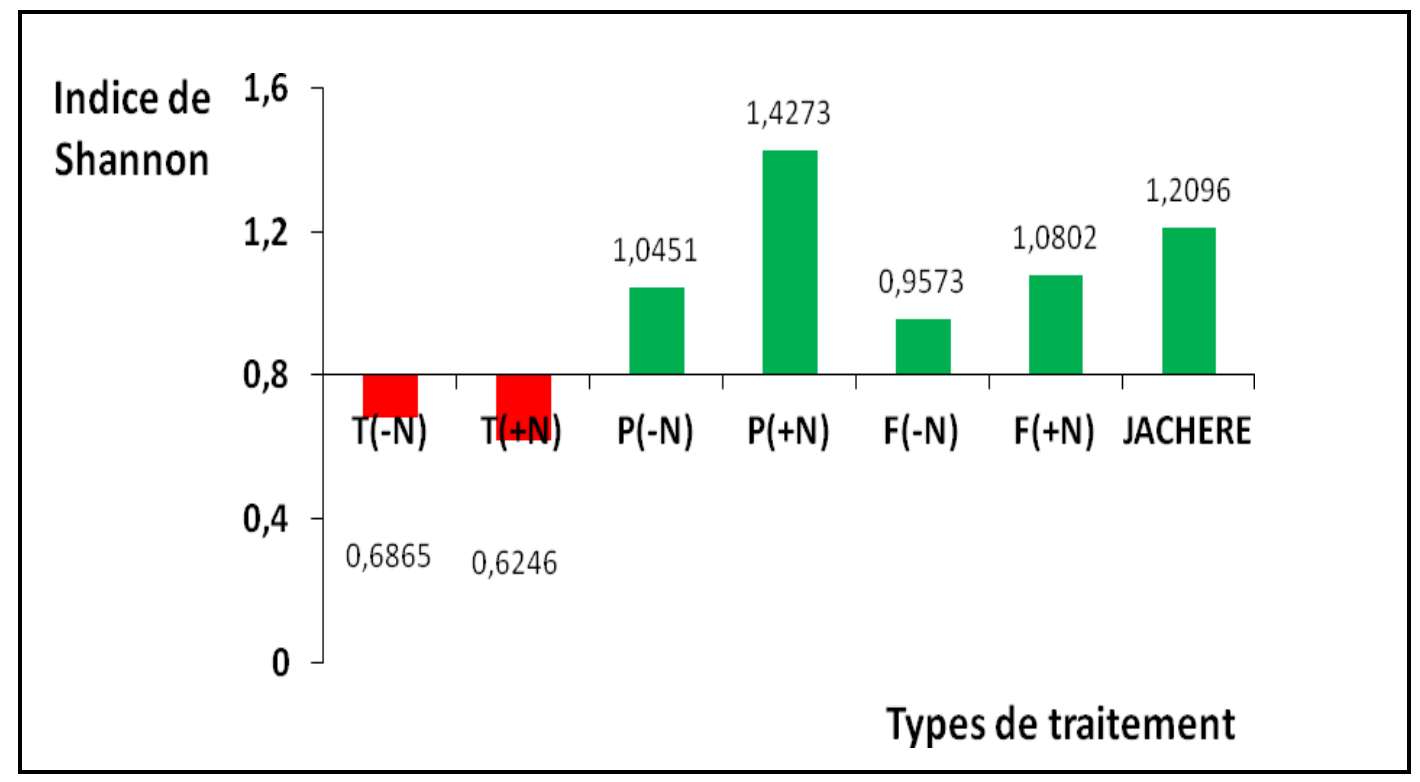

Figure 3: Position de l'Indice de Shannon des termites des différents traitements par rapport à la diversité moyenne.

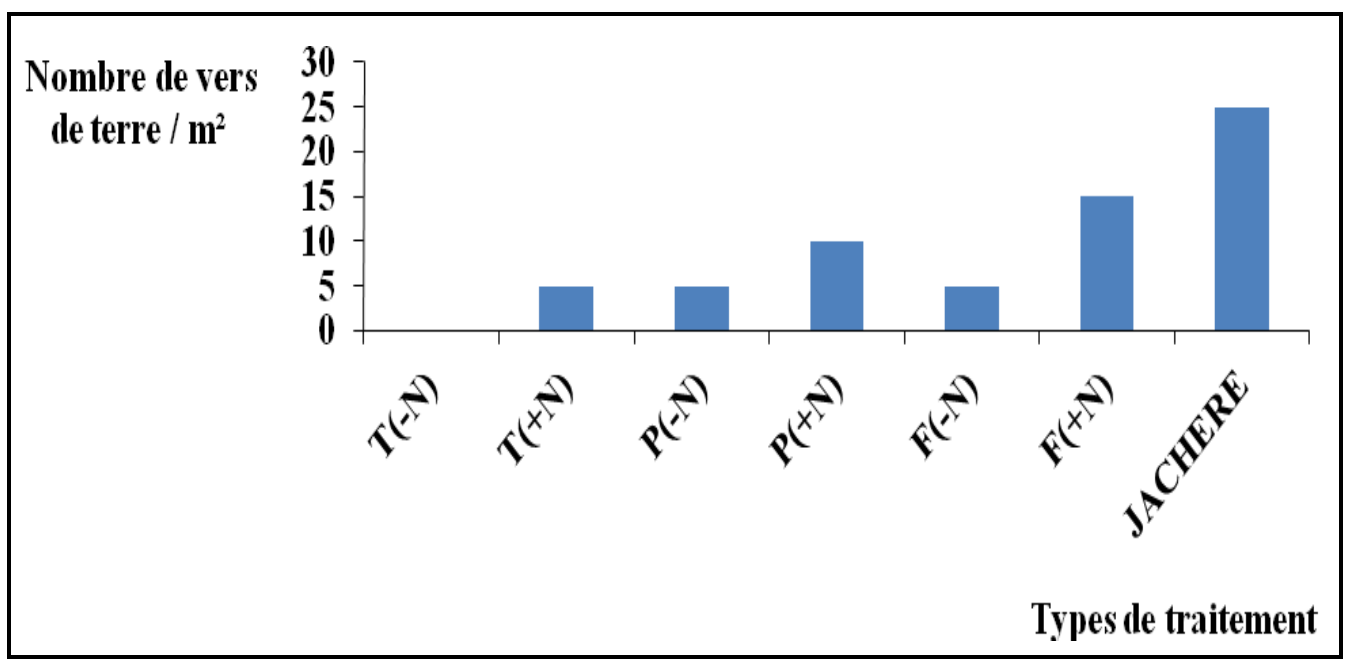

$\mathrm{T}(-\mathrm{N})$ : Témoin sans azote ; $\mathrm{T}(+\mathrm{N})$ : Témoin plus azote ; $\mathrm{F}(-\mathrm{N})$ : Fumier sans azote $; \mathrm{F}(+\mathrm{N})$ : Fumier plus azote ; $\mathrm{P}(-\mathrm{N})$ : Paille sans azote ; $\mathrm{P}(+\mathrm{N})$ : Paille plus azote.

Figure 4 : Nombre de vers de terre par unité de surface des différents traitements. 


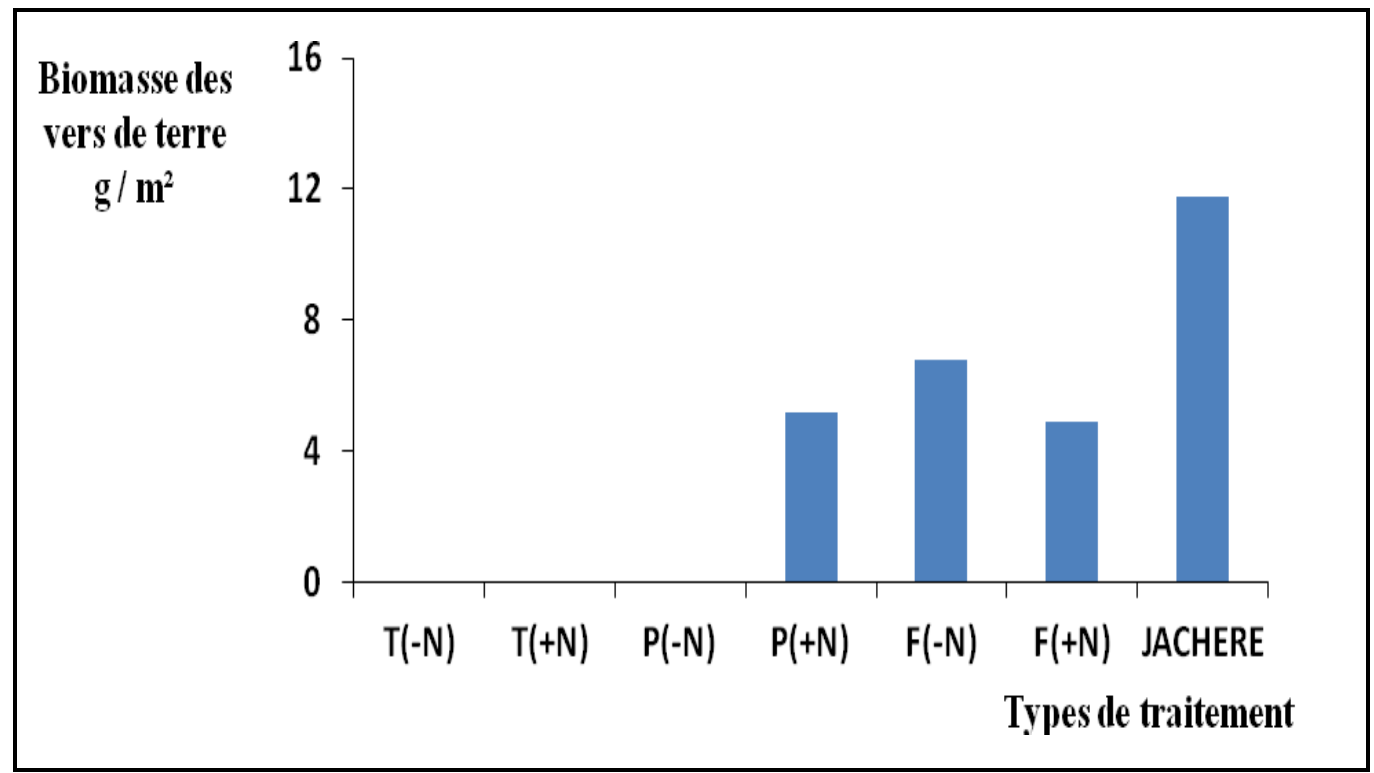

Figure 5: Biomasse des vers de terre par unité de surface des différents traitements.

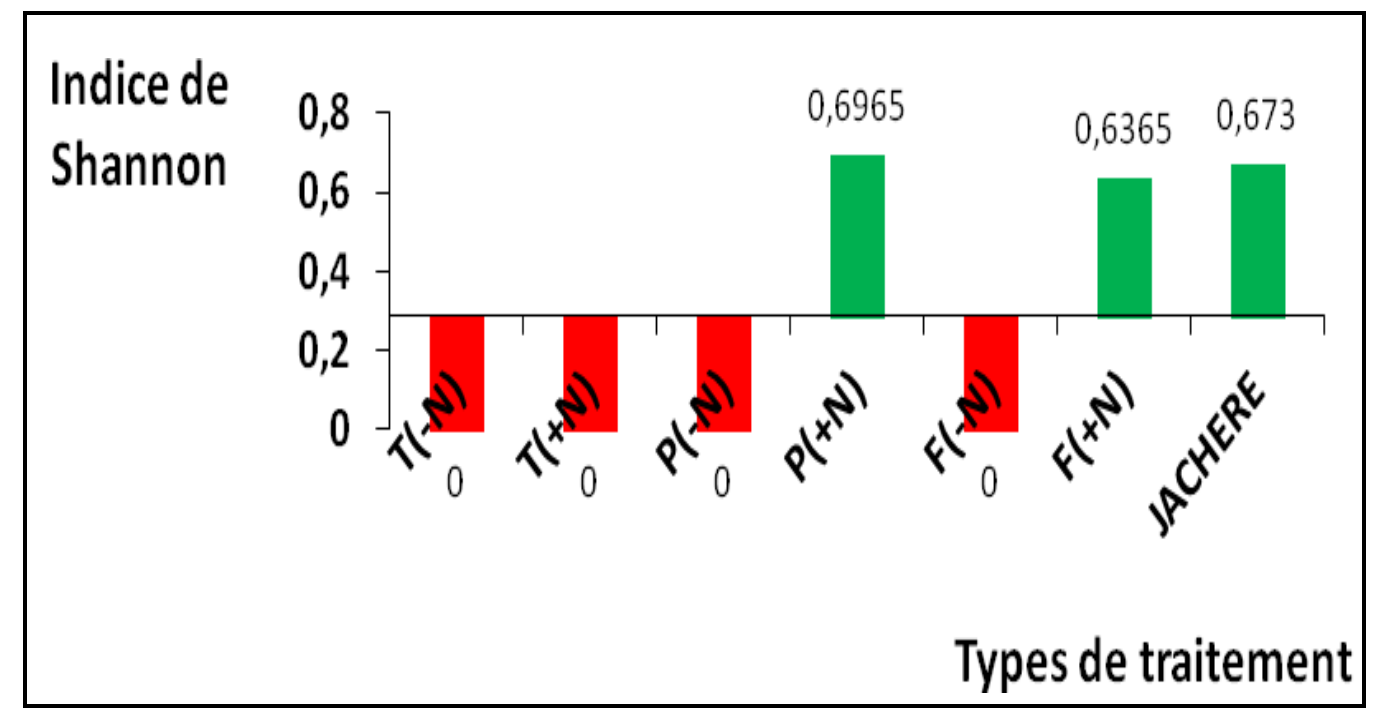

Figure 6: Position de l'Indice de Shannon des vers de terre des différents traitements par rapport à la diversité moyenne. 
Tableau 1: Individus, nombre, biomasse et Indice de Shannon des termites.

\begin{tabular}{|c|c|c|c|c|}
\hline $\begin{array}{l}\text { Types de } \\
\text { traitement }\end{array}$ & Individus & $\begin{array}{c}\text { Nombre } \\
\left(/ \mathbf{m}^{2}\right)\end{array}$ & $\begin{array}{c}\text { Biomasse } \\
\left(\mathbf{g} / \mathbf{m}^{2}\right)\end{array}$ & Indice de diversité \\
\hline & Termitidae (ailés) & $24 \pm 1,78$ & $0,05 \pm 0,0$ & \\
\hline $\mathrm{T}(-\mathrm{N})$ & Microtermes sp & $30 \pm 2,4$ & $0,04 \pm 0,0$ & 0,6865 \\
\hline \multirow[t]{3}{*}{$\mathrm{T}(+\mathrm{N})$} & Odontotermes magdalenae & $84 \pm 6,6$ & $0,17 \pm 0,0$ & \\
\hline & Termitidae (ailés) & $39 \pm 2,95$ & $0,06 \pm 0,0$ & 0,6246 \\
\hline & Microtermes $s p$ & $236 \pm 19$ & $0,22 \pm 0,0$ & \\
\hline \multirow{5}{*}{$\mathrm{P}(-\mathrm{N})$} & Termitidae (ailés) & $118 \pm 8,8$ & $0,06 \pm 0,0$ & 1,0451 \\
\hline & Odontotermes magdalenae & $270 \pm 22$ & $0,37 \pm 0,0$ & \\
\hline & Trinervitermes $s p$ & $34 \pm 2,3$ & $0,08 \pm 0,0$ & \\
\hline & Termitidae (ailés) & $206 \pm 16$ & $0,41 \pm 0,0$ & \\
\hline & Odontotermes magdalenae & $80 \pm 6,1$ & $0,17 \pm 0,0$ & 1,4273 \\
\hline \multirow[t]{3}{*}{$\mathrm{P}(+\mathrm{N})$} & Odontotermes $s p$ & $144 \pm 10,8$ & $0,13 \pm 0,0$ & \\
\hline & Microtermes sp & $55 \pm 4,0$ & $0,03 \pm 0,0$ & \\
\hline & Microtermes $s p$ & $15 \pm 0,9$ & $0,004 \pm 0,0$ & \\
\hline \multirow[t]{3}{*}{$\mathrm{F}(-\mathrm{N})$} & Termitidae (ailés) & $66 \pm 5$ & $0.17 \pm 0,0$ & 0,9573 \\
\hline & Odontotermes magdalenae & $60 \pm 4,3$ & $0,07 \pm 0,0$ & \\
\hline & Odontotermes magdalenae & $80 \pm 6,4$ & $0,18 \pm 0,0$ & \\
\hline \multirow[t]{3}{*}{$\mathrm{F}(+\mathrm{N})$} & Trinervitermes $s p$ & $80 \pm 6,1$ & $0,07 \pm 0,0$ & 1,0802 \\
\hline & Microtermes sp & $52 \pm 3,7$ & $0,04 \pm 0,0$ & \\
\hline & Trinervitermes $s p$ & $208 \pm 17$ & $0,60 \pm 0,0$ & \\
\hline \multirow[t]{3}{*}{ JS2 } & Macrotermes subhyalinus & $52 \pm 4$ & $0,74 \pm 0,1$ & 1,2096 \\
\hline & Amitermes stephensoni. & $72 \pm 5,2$ & $0,09 \pm 0,0$ & \\
\hline & Microcerotermes parvulus & $64 \pm 5,0$ & $0,18 \pm 0,0$ & \\
\hline
\end{tabular}

Tableau 2 : ANOVA des effets simples des types de traitements sur termites.

\begin{tabular}{lccccc}
\hline V1 & V2 & V3 & V4 & V5 \\
\hline P value & 0,083 & 0,141 & $\mathbf{0 , 0 2 7 *}$ & 0,072 & $\mathbf{0 , 0 1 1 *}$ \\
\hline *: Différence significative ; V1 Termidae (ailés); V2 & Trinertermes sp ; V3 Odontotermes magdalenae; \\
V4 Microtermes $s p ;$ V5 Odontotermes $s p$ &
\end{tabular}


M. TRAORE et al. / Int. J. Biol. Chem. Sci. 6(4): 1761-1773, 2012

Tableau 3: Individus, nombre, biomasse et Indice de Shannon des vers de terre.

\begin{tabular}{|c|c|c|c|c|}
\hline Types de traitement & Individus & $\begin{array}{c}\text { Nombre } \\
\left(/ \mathbf{m}^{2}\right)\end{array}$ & $\begin{array}{c}\text { Biomasse } \\
\left(\mathrm{g} / \mathrm{m}^{2}\right)\end{array}$ & Indice de diversité \\
\hline $\mathrm{T}(-\mathrm{N})$ & 0 & 0 & 0 & 0,000 \\
\hline $\mathrm{T}(+\mathrm{N})$ & Dichogaster affinis & $5 \pm 0,35$ & $0,055 \pm 0,0$ & 0,000 \\
\hline $\mathrm{P}(-\mathrm{N})$ & Dichogaster affinis & $5 \pm 0,38$ & $0,036 \pm 0,0$ & 0,000 \\
\hline $\mathrm{P}(+\mathrm{N})$ & $\begin{array}{l}\text { Dichogaster affinis } \\
\text { Millsonia inermis }\end{array}$ & $\begin{array}{l}5 \pm 0,34 \\
5 \pm 0,34\end{array}$ & $\begin{array}{c}0,065 \pm 0,0 \\
5,125 \pm 0,36\end{array}$ & 0,6965 \\
\hline $\mathrm{F}(-\mathrm{N})$ & Millsonia inermis & $5 \pm 0,38$ & $6,77 \pm 0,50$ & 0 \\
\hline $\mathrm{F}(+\mathrm{N})^{\circ}$ & $\begin{array}{l}\text { Juveniles } \\
\text { Millsonia inermis }\end{array}$ & $\begin{array}{c}10 \pm 0,74 \\
5 \pm 0,38\end{array}$ & $\begin{array}{c}1,14 \pm 0,0 \\
3,739 \pm 0,25\end{array}$ & 0,6365 \\
\hline Jachère & $\begin{array}{l}\text { Millsonia inermis } \\
\text { Dichogaster affinis }\end{array}$ & $\begin{array}{c}10 \pm 0,76 \\
15 \pm 1,2\end{array}$ & $\begin{array}{l}9,93 \pm 0,74 \\
1,822 \pm 0,0\end{array}$ & 0,6730 \\
\hline
\end{tabular}

Tableau 4: ANOVA des effets simples des types de fertilisation sur les vers de terre.

\begin{tabular}{llll}
\hline & V1 & V2 & V3 \\
\hline P value & 0,206 & 0,187 & 0,419 \\
\hline \multicolumn{2}{c}{ V1 Juveniles ; V2 Dichogaster affinis ; V3 Millsonia inermis }
\end{tabular}

V1 Juveniles ; V2 Dichogaster affinis ; V3 Millsonia inermis 


\section{DISCUSSION}

L'apport des matières organiques exogènes, représentées dans notre étude par la paille et le fumier, a montré un effet bénéfique pour les populations des deux groupes de macrofaune étudiés. Ces résultats sont conformes à ceux constatés dans de nombreux travaux antérieurs (Bachelier, 1978; Mboukou-Kimbatsa, 1997 ; Ouedraogo, 2004). Ces termites modifient leur environnement en remontant les particules du sous-sol vers la surface (Black et al., 1997; Deprince, 2003; Lavelle, 2006; Brussaard et al., 2007; Zaremski et al., 2009). L'incorporation de la paille a été nettement plus favorable aux termites par rapport au fumier. On émet l'hypothèse que la paille attirerait plus de termites que le fumier. Cet effet pourrait s'expliquer par leur teneur en cellulose. Analysant la teneur en cellulose de la paille et du fumier employé sur ce site, Hien (2004) a montré que la paille contenait $46 \%$ de cellulose et le fumier $8 \%$ de cellulose. A l'intérieur du même traitement, l'apport de l'azote s'est également accompagné d'une augmentation du nombre de macrofaune par unité de surface pour tous les traitements avec incorporation de fumier. Cette observation corrobore celles de Maldague (2003) et Jouquet et al. (2002) qui ont montré que l'apport d'azote dans le sol se traduit par un enrichissement en azote des microagrégats des termites par rapport au sol environnant. L'enfouissement de matières organiques exogènes a été également favorable aux populations de vers de terre. Les populations recensées sont des vers anéciques et endogés. $D$. affinis est un vers de terre géophage de faible profondeur (anécique) qui se nourrit essentiellement de terre prélevée dans l'horizon $0-10 \mathrm{~cm}$ et quelque fois plus profondément tandis que $M$. inermis est un vers géophage de grande profondeur (endogé) qui se nourrit de fractions organiques prélevées dans la terre à $30 \mathrm{~cm}$ de profondeur où ils vivent. Ce sont les vers de terre endogés qui dominent le plus souvent dans les sols tropicaux (Feller et al., 1993). A la différence des termites, c'est l'incorporation du fumier qui a été plus favorable à l'installation des vers de terre. L'apport d'azote s'est également traduit ici par une augmentation du nombre d'individus par unité de surface. Ce résultat corrobore ceux des travaux antérieurs qui ont montré qu'en présence d'une fertilisation azotée, les turricules s'enrichissent plus en azote par rapport au sol environnant car ils assurent une bonne fixation de l'azote (Dominguez et al., 2004 ; Ouédraogo et al., 2006). Ceci montre que les vers de terre sont très sensibles à la qualité et à la quantité de la matière qui exerce un effet favorable sur leur abondance. La jachère qui limite le site à l'est et au nord est une jachère à Adropogon gayanus (Hien, 2004). La macrofaune dans cette jachère a été plus diversifiée que dans le site de culture. De nombreux travaux antérieurs ont montré que la mise en culture des sols peut se traduire par une diminution de l'abondance et la diversité de la faune des sols (Bachellier, 1978 ; Feller et al., 1993 ; Dangerfield, 1990 ; Black et al., 1997; Mathieu, 2004) ou une modification de sa composition (Mboukou - Kimbatsa, 1997 ; Decaêns et al., 1999 ; Ruiz Camacho, 2004).

Les résultats montrent que les apports de matières organiques et d'une fertilisation minérale ont une influence sur la dynamique de macrofaune dans les sols étudiés. Ces résultats corroborent ceux de nombreux travaux antérieurs (Bachelier, 1978; Blanchart, 1990 ; Dover, 1991 ; MboukouKimbatsa, 1997 ; Decaëns, 1999 ; Ruiz, 2004). La faune du sol exerce un rôle prépondérant sur les propriétés et le fonctionnement du sol (Feller et al., 1993; Lavelle et al., 2006; Ouédraogo, 2004). On peut en conclure que les matières organiques 
et la fertilisation azotée permettent d'atténuer l'impact de la mise en culture sur la macrofaune en maintenant une diversité avec une meilleure répartition des espèces. Pour conserver la productivité des sols agricoles par la contribution bénéfique de la faune du sol, l'agriculture devrait promouvoir des pratiques qui conservent la biodiversité des invertébrés du sol qui sont représentés par les termites et les vers de terre sur le site étudié.

\section{Conclusion}

Les effets de la mise en culture des terres et de différentes pratiques culturales comme la fertilisation et l'incorporation de matières organiques exogènes ont été étudiés au cours de ce travail. L'apport de paille a attiré plus de termites par rapport au fumier et au traitement témoin. Les traitements avec apports de fumier et de paille ont été les plus diversifiés en termites et en vers de terre par rapport au témoin. A l'intérieur de chaque traitement, l'ajout de l'azote s'est traduit par une hausse du nombre d'individus aussi bien des termites que des vers de terre. Dans les jachères bordant le site de cultures, la diversité et la biomasse en macrofaune ont été plus importantes.

Nos résultats montrent que le maintien d'une diversité de la macrofaune du sol est possible sur les terres agricoles par la mise en œuvre de pratiques agricoles adéquates. Ces résultats peuvent servir de base pour une meilleure caractérisation de la dynamique de la macrofaune sous différentes pratiques culturales en prenant en compte l'influence des agents climatiques, édaphiques, chimiques ou biotiques très variés auxquels est soumis tout organisme qui vit dans le sol.

\section{REMERCIEMENTS}

Nous remercions Sanou Martin, technicien de Recherches à la Station de Recherches Agricoles de Saria pour son appui lors des travaux de terrain. Nous remercions également Dr Diallo Boukary Ousmane et Dr Guissou Tibi, Chargés de Recherches à l'Institut de l'Environnement et de Recherches Agricoles pour leur appui dans le traitement statistique des données.

\section{REFERENCES}

Anderson JD, Ingram JSI. 1993. Tropical Soil Biology and Fertility: A Handbook of Methods $\left(2^{\text {nd }}\right.$ edn). CAB International: Wallingford.

Bachelier G. 1978. La Faune du Sol, son Écologie et son Action. ORSTOM: Paris.

Blanchart E. 1990. Rôle des vers de terre dans la formation et la conservation de la structure des sols de la savane de Lamto (Côte d'Ivoire). Thèse de Doctorant, Université de Rennes-1, France, p. 263.

Black HIJ, Okwakol MJN. 1997. Agricultural intensification, soil biodiversity and agroecosystem function in the tropics: the role of the termites. Applied Soil Ecology, 6: $37-53$.

Bot JA, Nachtergaele OF, Young A. 2000. Land Resource Potentiel and Contraints at Regional and Country levels. FAO, World Soil Resources Report, Rome.

Bonzi M. 2002. Évaluation et déterminisme du bilan de l'azote en sols cultivés du centre Burkina Faso: Étude par traçage isotopique $15 \mathrm{~N}$ au cours d'essais en station et en milieu paysan. Thèse de Doctorat, INPL, Nancy, France, p.177.

Brussaard L, De Ruiter PC, Brown GG. 2007. Soil biodiversity for agricultural sustainability. Agriculture Ecosystems \& Environment, 121(3): 233-244.

Dangerfield JM. 1990. Abundance, biomass and diversity of soil macrofauna in savanna woodland and associated managed habitats. Pedobiologia, 34: 141150 . 
Decaëns T. 1999. Rôle fonctionnel et réponses aux pratiques agricoles des vers de terre et autres ingénieurs écologiques dans les savanes colombiennes. Thèse de doctorat, Université de Paris 06, Paris, France, p. 374.

Deprince A. 2003. La faune du sol: diversité, méthodes d'étude, fonctions et perspectives. Le Courrier de l'Environnement de l'INRA, 49: 19- 42.

Dominguez J, Bohlen JP, Parmeleew R. 2004. Earthworms increase nitrogen leaching to greater soil depths in row crop agroecosystems. Ecosystems, 7: 672-685.

Dover JW. 1991. The conservation of insects on arable farmland. In The Conservation of Insects and their Habitats, Collins NM, Thomas JA, (eds). London Academic Press: London; 294-315.

Feller C, Lavelle P, Albrecht A, Nlcolardot B. 1993. La jachère et le fonctionnement des sols tropicaux. Rôle de l'activité biologique et des matières organiques. Quelques éléments de réflexion. In $L a$ Jachère en Afrique de l'Ouest, Floret C, Serpantie G (eds). ORSTOM Collection Colloques et Séminaires: Paris, France; 15-32.

Fragoso C, Lavelle P. 1995. Are earthworms important in the decomposition of tropical litter ? In Soil Organisms and Litter Decomposition in the Tropics, Reddy MV (ed). Oxford \& IBH Publishing: Calcuta, New Delhi; 103-112.

Hien E. 2004. Dynamique du carbone dans un Acrisol ferrique du Centre Ouest Burkina: Influence des pratiques culturales sur le stock et la qualité de la matière organique. Thèse de Doctorat, Ecole Nationale Supérieure Agronomique de Montpellier, p. 140.

Jones TD, Eggleton P. 2000. Sampling termites assemblages in tropical forest: testing a rapid biodiversity assessment protocol. Journal of Applied Ecology, 37: 191- 203.

Jouquet P. 2002. Interactions matière organique-argiles dans les sols et nutriments assimilables par les plantes : les termites comme agents biologiques. Thèse de Doctorat, Université Paris 6, $140 \mathrm{p}$.

Lavelle P, Decaens M, Aubert M, Barot S, Bloiun M, Bureau F, Magerie P, Mora P, Rossi JP. 2006. Soil invertebrates and ecosystem services. European Journal of Soil Biology, 42(1): 3-15.

Lompo F. 2009. Effets induits des modes de gestion de la fertilité sur les états du phosphore et la solubililité des phosphates naturels dans deux sols du Burkina Faso. Thèse de Doctorat d'État ès-Sciences Naturelles, Université de Cocody, Côte d'Ivoire, p. 178.

Magurran AE. 1988. Ecological Diversity and its Measurements. Cambridge University Press: Great.

Maldague M. 2003. Les termites de la région de Bambesa (Uele, RDC) en relation avec la matière organique du sol. Bulletin de l'ANSD, 4: 7-75.

Marinissen JCY. 1992. Population dynamics of earthworms in a silt loam soil under conventional and "integrated" arable farming during two years with different weather patterns. Soil Biol. Biochem. 24: 1647-1654.

Mathieu J. 2004. Etude de la macrofaune du sol dans une zone de déforestation en Amazonie au Sud-Est, au Brésil, dans le contexte de l'agriculture familiale. Thèse de doctorat es Sciences en Ecologie, Université Pierre et Marie Curie - Paris 6, France, $237 \mathrm{p}$.

Mboukou-Kimbatsa IMC. 1997. Les macroinvertébrés du sol sous différents systèmes d'agriculture au Congo : Cas particulier de deux systèmes d'agriculture 
traditionnels (écobuage et brûlis) dans la vallée du Niari. Thèse de doctorat, Université Pierre et Marie Curie, Paris, France, p. 163.

Ouédraogo E, Mando A, Bruissaard L. 2006. Soil macrofauna affect nitrogen and water use efficiencies in semi-arid West Africa. European Journal of Soil Biology, 42(1): 275-277.

Ouédraogo E. 2004. Soil Quality Improvement for CropProduction in semi-arid West Africa; Tropical Ressources Mangement Papers No.51. Wageningen University and Rechearch Center, The Netherlands.

Ouédraogo S. 2005. Intensification de l'agriculture dans le plateau central du Burkina Faso: Une Analyse des possibilités à partir des nouvelles
Technologies. Thèse de PhD, Rijksuniversiteit Groningen, p. 336.

Ruiz Camacho N. 2004. Mise au point d'un système de bioindication de la qualité du sol basé sur l'étude des peuplements de macroinvertébrés. Thèse de Doctorat, Université de Paris 6, p. 327.

Sedogo PM. 1993. Evolution des sols ferrugineux lessivés sous culture : incidence des modes de gestion sur la fertilité. Thèse de Doctorat $3^{\text {ème }}$ cycle, Université Nationale de Côte d'Ivoire, p. 285.

Zaremski A, Fouguet D, Louppe D. 2009. Les Termites dans le Monde. CemagrefCirad-Ifremer-Inra: Paris, France. 